\title{
IS THE BILIARY MANOMETRIC PERFUSION TEST EFFECTIVE IN THE EVALUATION OF BALLOON DILATATION TREATMENT SUCCESS OF BENIGN BILIARY STRICTURES?
}

\author{
Rohit Philip Thomas ${ }^{\mathrm{a}}$, Martin Köcher ${ }^{\mathrm{a} *}$, Marie Cerna ${ }^{\mathrm{a}}$, Jiri Kozak ${ }^{\mathrm{a}}$, Stanislav Burval ${ }^{\mathrm{a}}$, \\ Roman Havlik
}

\author{
a Department of Radiology, Faculty of Medicine and Dentistry, Palacky University, I. P. Pavlova 6, 77520 Olomouc, Czech \\ Republic. \\ b Department of Surgery, University Hospital, I. P. Pavlova 6, 77520 Olomouc, Czech Republic.
}

Received: February 10, 2009; Accepted: April 18, 2009

Key words: Benign strictures/Biliary tract/Balloon dilatation/Percutaneous transhepatic drainage/Manometric test

Background: The success of balloon dilatation treatment of benign biliary strictures (BBS) is usually evaluated by 'clinical test'. For objective evaluation of treatment success, measurement of the pressure inside the biliary tree after treatment has been proposed.

Aim: The aim of this article is to assess the role of biliary manometric perfusion test (BMPT) in the evaluation of treatment success of BBS and to assess the long term biliary patency after evaluation by BMPT.

Materials and Methods: From February 2003 to January 2008, 12 patients (median age 53.5 years) after balloon dilatation treatment were evaluated by BMPT. BMPT was done after a mean of 3 dilatations over a mean period of 6.85 months. An intrabiliary pressure less than $20 \mathrm{~cm}$ of water during the test was considered as success threshold.

Results: The pressures during BMPT were less than $20 \mathrm{~cm}$ of water in 11 out of 12 patients. Subsequently the drain was removed and patients were followed up clinically. Two patients on follow-up required re-intervention 13 days and 11 months later. The remaining 9 patients had no evidence of biliary obstruction at a mean fellowing of 15.3 months. Using the statistical Kaplan-Meier analysis, the probability of biliary patency up to 9 months was $90.9 \%$ and at 1,2 and 3 years was $77 \%$.

Conclusion: BMPT is effective in the evaluation of treatment success of BBS. It is simple, cost effective, gives immediate results and helps us to determine the treatment end point.

\section{INTRODUCTION}

Benign biliary strictures are usually treated surgically or endoscopically. When surgical repair is impossible or endoscopic treatment is difficult, radiological percutaneous dilatation treatment is indicated. Percutaneous treatment includes initial drainage and dilatation of stricture by balloon angioplasty catheters of different diameters and long-term external-internal drainage ${ }^{1-4}$. When treated percutaneously, the typical sequence for determining treatment success and potential durability is the cholangiographic impression of the free flow of contrast medium across the treated stricture followed by the 'clinical test'. The clinical test involves leaving a small non functional $4 \mathrm{~F}$ safety catheter without side holes peripheral to or through the treated stenosis for a period of time (usually 7 to 10 days) and follow-up by liver function tests ${ }^{5}$. If the liver function tests are within the normal range, if the obstructive symptoms do not recur and bile leak from the percutaneous access is not seen, this suggests that the treated stricture will sustain prograde bile flow once the catheter is removed. Accordingly, the treatment is considered successful and the safety catheter is withdrawn. If the liver function tests are elevated or if there are obstructive symptoms in the form of bile leak from the percutaneous access, then another balloon dilatation and long term external internal drainage for another 3 mon- ths is done. For the objective evaluation of the treatment success, measurement of pressure inside the biliary tree (Biliary manometric perfusion test) has been proposed. We have earlier reported a case of biliary manometric perfusion test in a patient with benign biliary stricture and discussed the potential of the same in the treatment success evaluation ${ }^{5}$. The aim of this study was to assess the role of biliary manometric perfusion test (BMPT) in the evaluation of treatment success in a group of benign biliary stricture patients and to assess the long term biliary patency after treatment evaluation by manometric perfusion test.

\section{MATERIALS AND METHODS}

From February 2003 to January 2008, 12 patients ( 8 men, 4 women), with a median age of 53.5 years (range 41-80 years) had undergone biliary drainage and percutaneous dilatation treatment for benign biliary strictures. The causes for benign biliary strictures were strictures of hepaticojejunostomy in all patients. Indications for hepaticojejunostomy were injury to bile ducts during laproscopic cholecystectomy in 9 patients, duodenopancreatectomy in 2 patients and resection of benign tumor in one patient. Six patients had symptoms of obstructive jaundice or obstructive jaundice and cholangitis, two patients had symp- 
toms of biliary cirrhosis and four others had anicteric cholestasis. The clinical symptoms (obstructive jaundice, anicteric cholestasis, biliary cirrhosis or cholangitis) had appeared from 1 month to 8 years after the surgery.

After the initial transhepatic drainage, balloon dilatation and long term external internal drainage had been done for all patients according to the methodology percutaneous dilatation treatment described in literature ${ }^{1,5,6}$. Subsequently follow-up cholangiography usually after 6 months was performed. If there was evidence of uninterrupted flow of contrast medium across the treated stenosis, the manometric perfusion test was performed in the same session (Fig. 1). We followed the specifically reported and validated technique of Savader et $\mathrm{al}^{5-8}$. The preexisting internal external biliary catheter was exchanged for a 9 or $10 \mathrm{~F}$ sheath which was left peripheral to the treated stenosis and was connected by tubing and a three way stop cock to a manometer and a standard angiography injector. The tubing was flushed to clear any air and the manometer was zeroed at the level of mid axillary line. With the stop cock off the angiographic injector and on to the manometer, the baseline intrahepatic pressure was measured and recorded. The injector was loaded with contrast material diluted to $50 \%$ by normal saline. The dilution of contrast to $50 \%$ was done in order to approximate the viscosity of normal bile. Then the biliary tree was perfused through the side arm of the sheath with this diluted contrast at the following incremental rates: $2 \mathrm{ml} / \mathrm{min}$ for $5 \mathrm{~min}, 4 \mathrm{ml} / \mathrm{min}$ for $5 \mathrm{~min}, 8 \mathrm{ml} / \mathrm{min}$ for $5 \mathrm{~min}, 15 \mathrm{ml} / \mathrm{min}$ for $3 \mathrm{~min}$, and $20 \mathrm{ml} / \mathrm{min}$ for $2 \mathrm{~min}$. The continued adequacy of sheath position was reviewed fluoroscopically during each infusion. At the end of each perfusion the stopcock was taken off the angiographic injector and on to the manometer and the pressure in the biliary tree was measured and recorded. The advancement to the next infusion proceeded if the absolute intraductal pressure at the end of each perfusion was less than $20 \mathrm{~cm}$ of water and no complications or side effects (chills, pain or rigors) occurred. If all the pressure values during the perfusion test were less than $20 \mathrm{~cm}$ of water, the drain was removed and the patients were discharged. If pressure values were more than $20 \mathrm{~cm}$ of water, additional intervention was done by balloon dilatation and long term drainage for another 3 months ${ }^{5,6}$. Patients were followed up by gastroenterologists or surgeons for any symptoms of biliary obstruction. Periodic liver function tests were also done for evaluation of any signs of biliary obstruction.

\section{RESULTS}

The balloon dilatations of the strictures were done without any complications. An average of 3 dilatations (range 1 to 5) per patient was performed. The range of size of balloon used was from 5 to $12 \mathrm{~mm}$. Dilatation treatment was done over a mean period of 6.85 months (range 2.03 to 12.2 months). A normal interval of 3 months was decided in between sessions. However, if the patients returned for control cholangiographies in view of dislocated

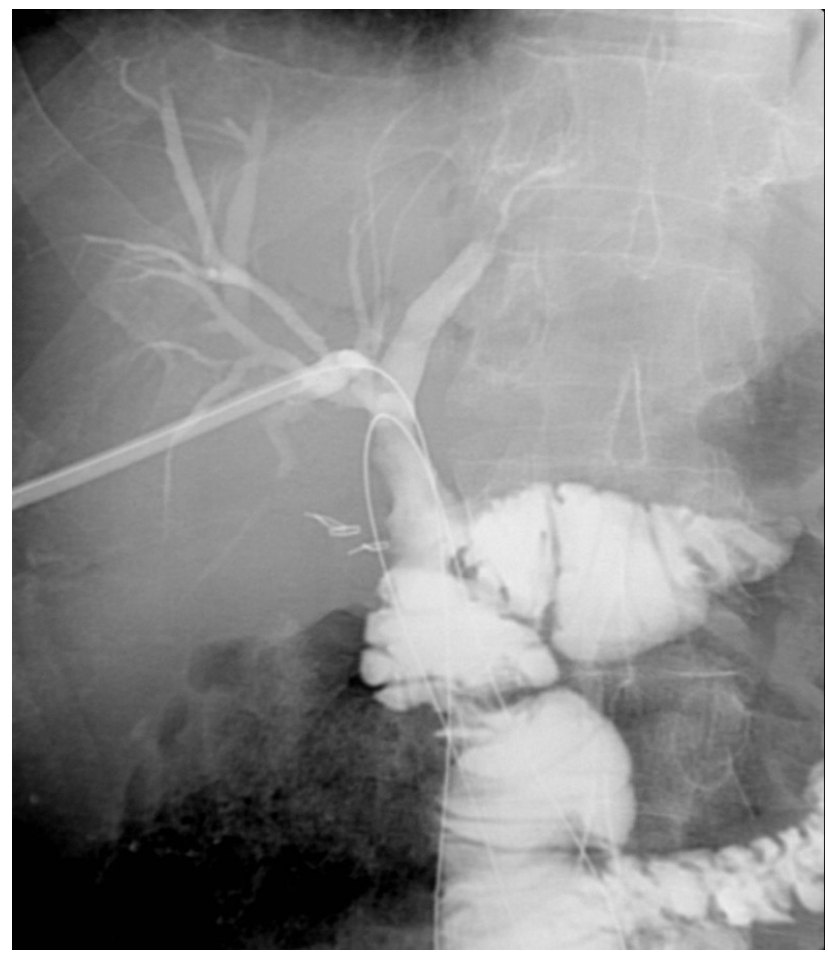

Fig. 1. A $9 \mathrm{~F}$ sheath left peripheral to the treated stenosis during manometric perfusion test showing decompressed bile ducts and free flow of contrast medium through the treated stenosis.

drains, we assessed the strictures and if needed we proceeded with balloon dilatation of the stenosis during the same session. The long term drainage and stricture remodelation was done with $12 \mathrm{~F}$ or $14 \mathrm{~F}$ catheters.

After the long term drainage (usually 6 months), follow up cholangiography was done. If there was evidence of free flow of contrast medium across the treated stenosis, manometric perfusion test was proceeded in the same session. The manometric test was done in all patients without any complications (pain, chills, fever and sepsis). In 11 out of 12 patients the pressure values during all the infusions of perfusion test were less than $20 \mathrm{~cm}$ of water. Subsequently the drain was removed in these 11 patients. The patients were followed up clinically and with liver function tests. The patient who had high pressure during the perfusion test was again treated with balloon dilatation and long term drainage. He required repeated balloon dilatations and an extra period of 15 months for long term drainage. After the prolonged long term drainage, on control cholangiography there was free flow of contrast medium across the treated stenosis. For this reason he was again subjected to manometric perfusion test and the pressures during the perfusion test were less than $20 \mathrm{~cm}$ of water. The drain was removed and upto date he has no symptoms of biliary obstruction. Two other patients out of the 11 patients who had undergone manometry and drain removal required re-intervention at 13 days and 11 months later on follow up. These two patients had also undergone another balloon dilatation and long term drainage for more time. One of these two patients 


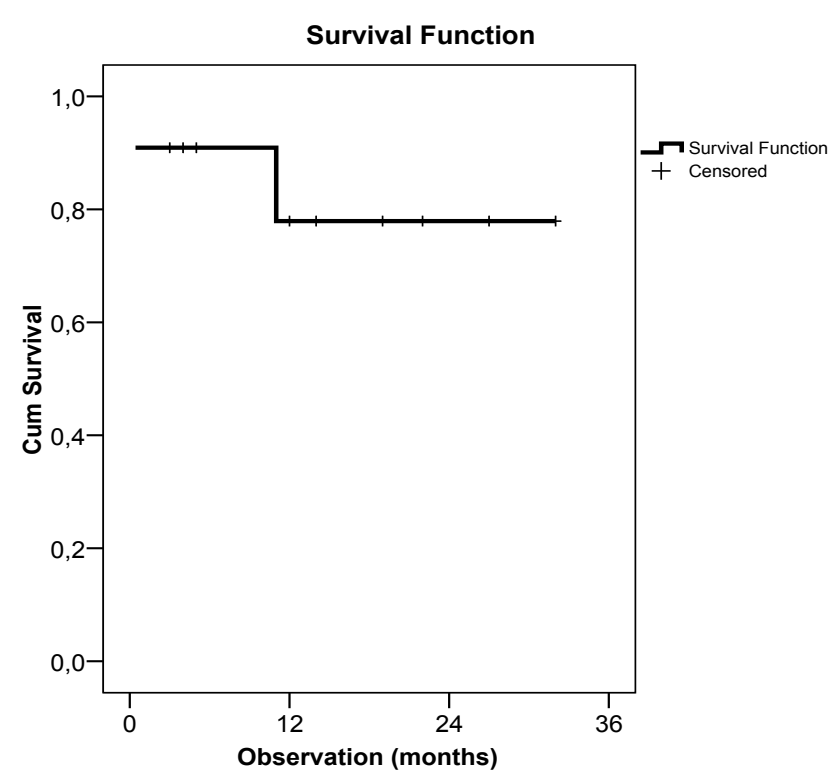

Fig. 2. Kaplan Meier analysis for long term biliary patency.

who required re-intervention 13 days later was diagnosed to have associated secondary biliary cirrhosis and is still undergoing long term drainage. The other patient who was again treated with balloon dilatation required a period of 9 months for long term drainage. This patient too after prolonged long term drainage was subjected to the manometric perfusion test again. The pressures during the infusions were less than $20 \mathrm{~cm}$ of water. Subsequently the drain was removed and on follow up till date he has no recurrence of biliary obstruction. The remaining $9 \mathrm{pa}-$ tients who were followed up after removal of drain remain symptom free at a mean follow-up of 15.3 months (range 3 to 32.3 months). One patient died 3 months later for reasons unrelated to our intervention (respiratory failure and sepsis). Until his death, he had no evidence of biliary obstruction. Using the statistical Kaplan-Meier analysis, the probability of biliary patency at 3,6, 9 months was $90.9 \%$ and at 1 year, $2 \& 3$ years was $77 \%$ (Fig. 2).

\section{DISCUSSION}

The decision of end points of radiological percutaneous treatment of benign biliary strictures is often a great challenge to the treating physician. Some of these patients require repeated balloon dilatations and a prolonged period of drainage and stricture remodelation. The decision of adequacy of treatment is rather difficult. Often we have cholangiographic evidence of free flow of contrast material through the treated stenosis during control cholangiography after treatment. The liver function tests during the clinical test may be within the normal range but on follow up they may again present with restenosis later and need a completely new transhepatic puncture and additional balloon dilatation treatment. It is often difficult to decide when to stop the treatment or the treatment end point.
Accordingly, measuring the functional parameters of the treated stenosis is proposed and reported as a better objective evaluation of the treatment success ${ }^{5-8}$. The long term success of the treated stenosis depends on the functional status of the treated stenosis. The functional status of treated biliary stenosis depends on the ability to sustain free flow and pressure. Similar to urologic perfusion studies, manometry recordings with perfusion challenges in the biliary tree have been reported in a variety of biliary pathologies 9 . A relation of intrabiliary pressure to poor duct caliber has also been demonstrated. The measurement of pressure after the radiological percutaneous treatment is a direct measurement of functional status of the treated stricture. It helps us to ensure the cholangiographic evidence of free flow across the treated stenosis more objectively. Similarly manometry has been long used as a pre and post operative tool for assessing the results of biliary surgery, endoscopic sphinterotomy and other biliary conditions ${ }^{10-13}$.

After trying the manometric perfusion test to evaluate the treatment success in our patient group, the results were thoroughly analyzed. Out of 11 patients in whom the manometric perfusion test was completed, nine patients remain symptom free at mean follow up period of 15.3 months. From this it can be concluded that the manometric perfusion test is successful in evaluating the dilatation treatment of benign biliary strictures. From our patient group, the patient who required secondary intervention 13 days after the manometric perfusion test, was diagnosed to have secondary biliary cirrhosis. The inadequacy of the treatment may be correlated to the underlying liver disease. Apart from this patient, only one out of 11 patients returned with symptoms of biliary obstruction after the manometric perfusion test. And the long term biliary patency with Kaplan-Meier analysis in our group is $77 \%$ at the third year. From this it can be concluded that manometric perfusion test is effective in the evaluation of treatment success from the long term point of view. There are very few studies in the literature that deal with measurement of pressure after dilatation treatment of benign biliary strictures. Our results are comparable to the studies described in literature. Savader et al reported that manometric perfusion is effective in treatment evaluation and has the same efficacy as the clinical test in evaluating treatment success ${ }^{7,8}$. With the same efficacy as the clinical test, these authors preferred manometric perfusion test for its simplicity and immediate results.

The manometric perfusion test is simple, provides immediate results and is cost effective. It is a single session test unlike the clinical test which takes a minimum of 7 days. In terms of patients' and physicians' time and resources, the perfusion test offers the patient the advantage of discontinuing biliary intubation 1 or 2 weeks earlier than with the clinical test. Further biliary intubation with small catheter during clinical test carries a risk of encrustation with bile salts, obstruction, interim catheter dislodgement and cholangitis ${ }^{14}$. The manometric perfusion test is cost effective as money is saved for the safety catheter and other biochemical tests before catheter 
extraction which includes liver function tests. The efficacy of the perfusion test lies not only in its advantage of predicting the post treatment patency but also in identifying potential treatment failures thereby reducing the number of patients who might later develop strictures after the treatment is completed ${ }^{7}$.

The results of our study show that manometric perfusion test is effective in the treatment evaluation of benign biliary strictures. Our study is naturally limited by the small size of the group and the short period of follow up. The recurrence of biliary stricture has been reported to occur as late as 20 years $^{15}$. Therefore these patients should be followed up for a longer period of time. However, the results are promising enough that manometric perfusion test has become our rourine after the diletation treatment of all benign biliary strictures.

\section{CONCLUSION}

The manometric perfusion test is effective in the treatment success of benign biliary strictures. It is simple, cost effective, provides immediate results and enables us to decide treatment end points more effectively.

\section{ACKNOWLEDGEMENTS}

This work was supported by the grant IGA MZ CR No. NS 10274-3.

\section{REFERENCES}

1. Kocher M, Cerna M, Havlik R, Kral V, Gryga A, Duda M Percutaneous treatment of benign bile duct strictures. Eur J Radiol. 2007; 62(2):170-74.
2. Laasch HU, Martin DF. Management of benign biliary strictures. Cardiovascular and Interventional Radiology 2002; 25:457-66.

3. Misra S, Melton GB, Geschwind JF, Venbrux AC, Cameron JL, Lilleomoe KD. Percutaneous management of bile duct strictures and injuries associated with laparoscopic cholecystectomy: a decade of experience. J Am Coll Surg. 2004;198(2):218-26.

4. Vos PM, van Beek EJ, Smits NJ, Rauws EA, Gouma DJ, Reeders JW.Percutaneous balloon dilatation for benign hepaticojejunostomy strictures. Abdom Imaging 2000;25:134-8.

5. Thomas RP, Kocher M, Cerna M, Kozak J, Havlik R. Biliary manometric perfusion test in the evaluation of treatment evaluation of benign biliary stricture- A case report. Biomed Pap Med Fac Univ Palacky Olomouc Czech Repub.2008; 152(2):293-97.

6. Thomas RP, Kocher M. Percutaneous treatment of benign biliary strictures and biliary manometric perfusion test. Biomed Pap Med Fac Univ Palacky Olomouc Czech Repub. 2007; 151(1):85-90.

7. Savader SJ, Cameron JL, Lillemoe KD, Lund GB, Mitchell SE, Venbrux AC.The biliary manometric perfusion test and clinical trial - long-term predictive value of success after treatment of bile duct strictures: ten-year experience. J Vasc Interv Radiol 1998; 9(6):976-85

8. Savader SJ, Cameron JL, Pitt HA, et al. Biliary manometry versus clinical trial:value as predictors of success after treatment of biliary tract strictures. J Vasc Interv Radiol 1994; 5(5):757-63.

9. VanSonnenberg E, Ferrucci JT Jr, Neff CC, Mueller PR, Simeone JF,Wittenberg J. Biliary pressure : Manometric and perfusion studies at percutaneous transhepatic cholnagiography and percutaneous drainage. Radiology 1983; 148:41-50.

10. White TT, Waisman H, Hopton D, Kavlie H. Radiomanometry, flow rates and cholangiography in the evaluation of common bile duct disease: a study of 220 cases. Am J.Surg 1972; 123:73-79.

11. Hopton D. Common bile duct perfusion combined with operative cholangiography. Br J.Surg 1978; 65:852-854.

12. Mallet-Guy P, Rose JD. Per operative manometry and radiology in biliary tract disorders. Br J.Surg 1956; 44:55-68.

13. Bar-Meir S, Geenen JE, Hogan WJ, Dodds WJ, Stewart ET, Arndorfer RC. Biliary and pancreatic duct pressures by ERCP manometry in patients with suspected papillary stenosis. Dig Dis Sci 1979; 24: 209-213.

14. Haskal ZJ, Brown RS Jr. Role of Biliary stress manometry after Biliary stricture dilation in Living Donor Liver Transplant Recipients. J Vasc Interv Radiol 2008; 19 ( 2 Pt 1): 216-19.

15. Pitt HA, Miyamoto T, Parapatis SK, Tompkins RK, Longmire WP Jr. Factors influencing outcome in patients with post operative biliary strictures. Am J.Surg 1982; 144:14-21. 